\title{
Instrumentos de avaliação da sexualidade em homens e mulheres após a lesão medular: uma revisão sistemática
}

\author{
Assessment instruments of sexuality in men and women after \\ spinal cord injury: a systematic review
}

Ana Carolina Monteiro Lessa de Moura ${ }^{1}$, (D) Pollyana Helena Vieira Costa ${ }^{1}$, (D)anaine Cunha Polese ${ }^{1}$

\section{RESUMO}

A disponibilidade de instrumentos para avaliação da saúde sexual é muito importante para uma boa prática clínica, pois permite que os profissionais da área da saúde avaliem e intervenham de forma eficaz, atingindo os melhores padrões de avaliação e intervenção, e assim, melhorem a vida sexual dessa população. Objetivo: Revisar sistematicamente os instrumentos de avaliação da sexualidade em homens e mulheres após a lesão medular disponíveis na literatura, em relação ao seu uso e propriedades de medida. Método: Trata-se de uma revisão sistemática da literatura das bases de dados Pubmed, Lilacs, Scielo e Cinahl, de todos os estudos até maio de 2019, em que a estratégia de busca consistiu na utilização das palavras-chave: sexuality, spinal cord injury, evaluation, assessment e questionnaires. Os artigos identificados pela estratégia de busca inicial foram avaliados conforme critérios de inclusão pré-estabelecidos. Os desfechos foram mecanismos sexuais (excitação, ejaculação, ereção e orgasmo), desejo, satisfação, frequência sexual, ajuste, educação sexual e relação com o parceiro. Os dados foram organizados em tabelas foram apresentados através de uma análise descritiva dos resultados dos estudos incluídos. Resultados: Dezoito estudos atenderam aos critérios de elegibilidade e foram incluídos. Foram encontrados sete instrumentos validados para avaliação da sexualidade em indivíduos com lesão medular, nove genéricos e doze instrumentos para avaliação de fatores possivelmente relacionados a sexualidade. Dois avaliaram as propriedades de medida, sendo que em ambos foram adequadas. Conclusão: Esta revisão identificou um total de 28 instrumentos que foram utilizados na avaliação da sexualidade em indivíduos com lesão medular, entretanto, apenas sete desses instrumentos foram validados.

Palavras-chave: Traumatismos da Medula Espinal, Avaliação da Deficiência, Sexualidade, Aconselhamento Sexual

\section{ABSTRACT}

The availability of tools for sexual health assessment is very important for good clinical practice as it enables health professionals to evaluate and intervene effectively, achieving the best standards of assessment and intervention, and thus improving sex life of this population. Objective: To systematically review the instruments for assessing sexuality in men and women after spinal cord injury available in the literature regarding their use and measurement properties. Methods: This is a systematic literature review of the Pubmed, Lilacs, Scielo and Cinahl databases from all studies up to May 2019, in which the search strategy consisted of using the keywords: sexuality, spinal cord injury, evaluation, assessment and questionnaires. The articles identified by the initial search strategy were evaluated according to the pre-established inclusion criteria. Outcomes were sexual mechanisms (arousal, ejaculation, erection and orgasm), desire, satisfaction, sexual frequency, adjustment, sex education and relationship with the partner. Data were organized into tables were presented through a descriptive analysis of the results of the included studies. Results: Eighteen studies met the eligibility criteria and were included. We found seven validated instruments for assessing sexuality in individuals with spinal cord injury, nine generic and twelve instruments for assessing factors possibly related to sexuality. Two evaluated the measurement properties, and both were adequate. Conclusion: This review identified a total of 28 instruments that were used to assess sexuality in individuals with spinal cord injury, however, only seven of these instruments were validated.

Keywords: Spinal Cord Injuries, Disability Evaluation, Sexuality, Sex Counseling 


\section{INTRODUÇÃO}

A lesão medular é caracterizada por qualquer comprometimento que acometa a medula espinhal, trazendo déficits na função motora, sensitiva, visceral e sexual do indivíduo. ${ }^{1}$ Dessa forma, quando o indivíduo possui lesão medular, alguns mecanismos sexuais ficam comprometidos, tais como a capacidade erétil, de ejaculação, da qualidade do sêmen, orgásmica, de fertilidade, além das alterações psicológicas. ${ }^{2}$

A sexualidade é um termo abrangente que envolve aspectos físicos e emocionais, enquanto a função sexual geralmente se refere aos aspectos fisiológicos das respostas sexuais. ${ }^{3} \mathrm{~A}$ função sexual pode ser afetada por problemas físicos causados por um distúrbio neurológico, como espasticidade, dor, controle intestinal e vesical, mas também por questões psicológicas. ${ }^{4}$

Dessa forma, ao avaliar a função sexual de pacientes com lesão medular, todos esses fatores devem ser levados em consideração. ${ }^{5} \mathrm{~A}$ atividade sexual é classificada como a primeira prioridade para a recuperação da função em paraplégicos e a segunda entre aqueles com tetraplegia, depois de recuperar a função de braço e mão. 6,7

Sendo assim, é importante estabelecer a compreensão dos mecanismos sexuais e de seus correlatos, com fatores demográficos relacionados a lesão medular, saúde geral e fatores de qualidade de vida. ${ }^{8}$

A disfunção sexual nesses indivíduos pode ser decorrente de vários fatores, como alteração da sensibilidade, distúrbios eréteis e autonômicos, problemas psicológicos, difícil posicionamento para a relação sexual e complicações, como dor neuropática, incontinência urinária e fecal e úlceras de pressão. ${ }^{6}$

A avaliação dos aspectos da sexualidade nessa população é importante para que os profissionais da área da saúde avaliem as limitações e intervenham de forma eficaz, atinjam os melhores padrões de prática e assim, melhorem a qualidade de vida dos indivíduos com lesão medular. ${ }^{9}$ Porém, observa-se na literatura que a maioria dos estudos avaliam a sexualidade na lesão medular de forma qualitativa ou com instrumentos não validados.

\section{OBJETIVO}

O objetivo desta revisão, foi analisar sistematicamente os instrumentos de avaliação da sexualidade em homens e mulheres após a lesão medular disponíveis na literatura, em relação ao seu uso e propriedades de medida.

\section{MÉTODOS}

Trata-se de uma revisão sistemática da literatura. A busca bibliográfica foi realizada por um pesquisador e checada por um segundo pesquisador nas seguintes bases de dados: Pubmed, Lilacs, Scielo e Cinahl (até maio de 2019), sem restrições de língua ou data.

A estratégia de busca inicial consistiu na utilização das palavraschave: sexuality, spinal cord injury, evaluation, assessment e questionnaires.

Critérios de inclusão: estudos descritivos, transversais, quaseexperimentais, ensaios clínicos, estudos metodológicos e relatos de caso, amostra composta por homens e mulheres com lesão medular (de qualquer etiologia), com idade igual ou superior a 18 anos de idade, estudos que utilizaram instrumentos de avaliação da sexualidade (questionários e/ou inventários) aplicado em homens e/ou mulheres com lesão medular. Critérios de exclusão: revisões sistemáticas.

Foram extraídas informações sobre a metodologia dos estudos (design, participantes, intervenção medidas de desfecho) e de seus resultados obtidos por dois pesquisadores independentes.

Quando o estudo não apresentou dados suficientes, os autores foram contatados via e-mail. Os dados coletados foram apresentados através de uma análise descritiva dos resultados dos estudos incluídos.
Para avaliar o risco de viés entre os estudos incluídos, foram analisados individualmente os desfechos, metodologia utilizada e propriedades de medidas do desenvolvimento de cada instrumento por dois pesquisadores, que realizaram a avaliação de forma independente.

\section{RESULTADOS}

A estratégia de busca inicial, utilizando as palavras-chave: sexuality, spinal cord injury e evaluation, considerando as bases de dados analisadas (Pubmed, Scielo, Lilacs e Cinahl), retornou 122 títulos e resumos. Após a avaliação de títulos e resumos, restaram 18 estudos potencialmente elegíveis. Após a leitura na íntegra dos estudos, foi encontrado um total de 11 artigos que preencheram os critérios de inclusão e foram utilizados na presente revisão. Além disso, foram incluídos 7 estudos através da busca manual, totalizando na presente pesquisa um total de 18 estudos incluídos (Figura 1).

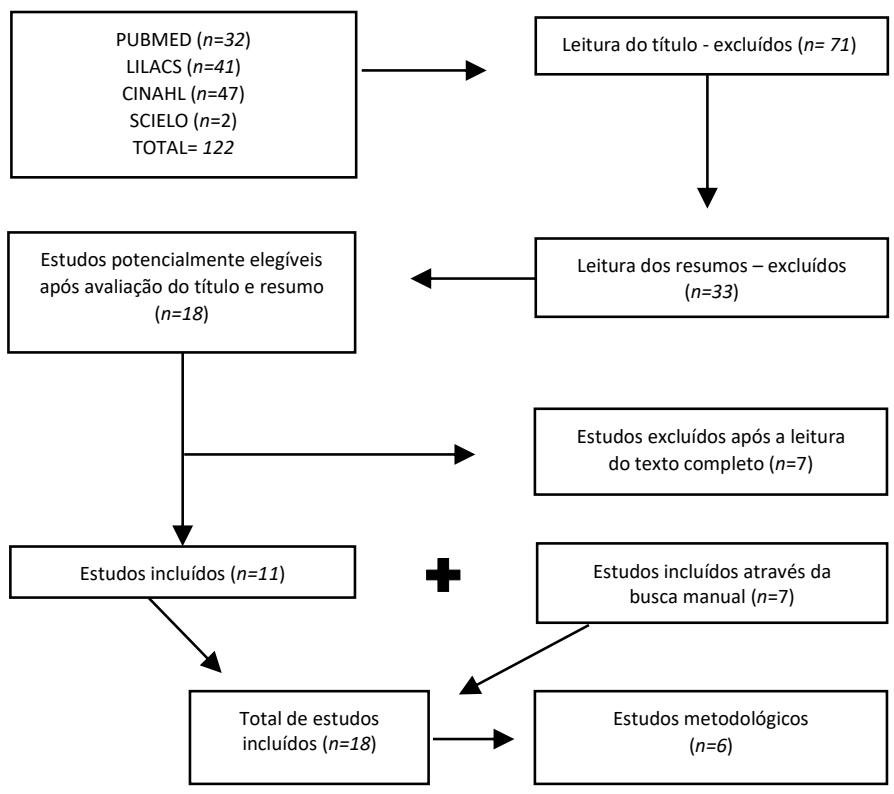

Figura 1. Fluxograma de inclusão e exclusão dos estudos

Dentre os 18 estudos incluídos, 9 eram estudos transversais, 2 casos controle, 1 descritivo e 6 metodológicos. O número de participantes envolvidos em cada estudo variou entre 30 e 1039 participantes, com idade média variando entre 18 e 60 anos (Quadro 1).

Os instrumentos para avaliação de fatores que podem estar relacionados a sexualidade utilizados em seis estudos ${ }^{8,10-14}$ foram: LISAT-8, EuroQoL, General Health Questionnaire (GHQ-12), The Hospital Anxiety and Depression Scale (HADS), The Rosenberg SelfEsteem Scale (RSES), The Sexual Health Evaluation Scale (SHES), Emotional Quality of the Relationship (EQR) scale, Sickness Impact Profile (SIP), Mood Adjective Check List (MACL), Spinal Functional Ability Scale (S-FAS), International SCl Basic QoL Data Set, Body Satisfaction Scale (BSS).

Os instrumentos genéricos, ou seja, que avaliam sexualidade, mas não especificamente na população com lesão medular, utilizados em sete estudos ${ }^{10,12-17}$ foram: International Index of Erectile Function-5 (IIEF-5), Sexual Health Evaluation Scale (SHES), Body Satisfaction Scale (BSS), Sexual Activity and Satisfaction Scale (SAS), Sexual Behavior Scale (SB), Sexuality and sexual life Spinal Cord Injury Women Questionnaire (SCIWQ), Sexual Interest Activity and Satisfaction scale (SIAS), Integrated index of sexual function (IISF), Sexual Interest and Satisfaction scale (SIS). Esses instrumentos abordam desfechos como: mecanismos sexuais (excitação, ejaculação, ereção e orgasmo), desejo, satisfação, frequência sexual, ajuste, educação sexual e relação com o parceiro. 
Os instrumentos específicos validados para avaliação da sexualidade em indivíduos com lesão medular utilizados em seis estudos 6,7,18-21 foram: Male Sexual Quotient (MSQ), Sexual Health Inventory for Men (SHIM), Sexual Adjustment Questionnaire (SAQ), Sexual Attitude and Information Questionnaire (SAIQ), "Perceived Sexual Distress Scale" (PSDS), "International SCI male sexual function" e "female sexual and reproductive function" (SR-iSCl-sexual function), Questionário de Sexualidade Humana na Lesão Medular (QSH-LM) e Sexual health measures (SHM). Nesses estudos, foram investigadas as seguintes propriedades de medida: validade e confiabilidade (Quadro 2).

\section{DISCUSSÃO}

Dentre os 19 estudos incluídos nessa revisão, todos avaliaram a sexualidade de indivíduos com lesão medular através de algum questionário ou entrevista, seja já validado (7 estudos) para essa população ou desenvolvido pelos próprios autores dos estudos ( 8 estudos).

Seis estudos metodológicos avaliaram as propriedades de medida de instrumentos de avaliação da sexualidade. Dentre os desfechos avaliados por cada um dos instrumentos, pode-se destacar: questões fisiológicas e específicas da sexualidade, como excitação, ereção, ejaculação e orgasmo, mas também seus correlatos, como fatores demográficos relacionados a lesão medular, estilo de vida, saúde mental e qualidade de vida.

As amostras dos estudos foram heterogêneas em relação ao gênero dos indivíduos incluídos. A população mais prevalente foi de apenas homens ${ }^{1,6,8,10,15,22}$ ou de homens e mulheres, sendo nesse caso a porcentagem de mulheres menor.7,12,13,16-21

Essa prevalência de homens pode ser decorrente da predominância masculina entre os indivíduos traumaticamente lesados e também pelo fato de que a disfunção sexual feminina pode ser considerada menos problemática para as mulheres do que para os homens, pois ainda existe a crença de que as mulheres possuem um papel passivo na relação sexual. ${ }^{4}$

É possível identificar na literatura uma lacuna entre os estudos que investigam a sexualidade em mulheres com lesão medular. $\mathrm{Na}$ presente revisão, apenas três estudos ${ }^{11,14,23}$ investigaram a sexualidade exclusivamente de mulheres. Os problemas mais prevalentes relatados pelas mulheres são acidentes urinários e intestinais durante o ato sexual e relação com o parceiro. ${ }^{9}$

Os instrumentos disponíveis na literatura para avaliação exclusivamente de mulheres, ainda não são validados para essa população e em sua maioria são questionários desenvolvidos pelos próprios autores, mas podem ser úteis para investigação dessa população.

Dois estudos desenvolveram questionários auto-administrados que permitem colher informações relacionadas a atividade sexual, natureza da disfunção sexual e aconselhamento sexual. ${ }^{14,23}$ Além desses, existe o questionário SCIWQ (Sexuality and sexual life Spinal Cord Injury Women Questionnaire) que também avalia os aspectos citados acima. ${ }^{11}$

"A disfunção sexual, que inclui disfunção erétil e ejaculatória, transtorno orgásmico, desejo sexual hipoativo e distúrbios de dor sexual são altamente prevalentes em homens". ${ }^{24}$ Sendo assim, é essencial a utilização de instrumentos específicos para homens que avaliem essas variáveis. Foram encontrados na literatura ${ }^{6,15}$ dois instrumentos com esse fim: International Index of Erectile Function (IIEF-5) e Sexual Health Inventory for men (SHIM).

Alguns estudos $8,10,12-16$ incluíram instrumentos de avaliação de fatores não específicos da sexualidade, mas que estão intimamente relacionados a ela. Dentre os instrumentos, pode-se citar: EuroQol, General Health Questionnaire (GHQ-12), The Hospital Anxiety and Depression Scale (HADS), Rosenberg Self-Esteem Scale (RSES), Sickness Impact Profile (SIP), Mood Adjective Check List (MACL), Spinal Functional Ability Scale (S-FAS) e International SCI Basic QoL Data Set.
Os desfechos investigados por esses instrumentos estão descritos no Quadro 1.

Sabe-se que os problemas físicos e fisiológicos relacionados a sexualidade interferem no estado de saúde em geral e vice-versa. Dessa forma, investigar sobre a qualidade de vida do paciente, avaliar o bem-estar geral e saúde mental (ansiedade e transtornos depressivos) também é importante quando se quer abordar a complexidade da sexualidade, pois a função sexual sofre interferência direta de questões psicológicas, autoestima e relacionamento. ${ }^{4}$

Os questionários utilizados para avaliação da sexualidade, podem ser feitos em formato de autoadministração, em que o paciente mesmo responde o questionário entregue, ou em formato de entrevista, onde o profissional da área da saúde faz as perguntas para o paciente. Dentre os estudos incluídos na presente revisão, apenas três ${ }^{17,20,23}$ utilizaram instrumentos feitos em formato de entrevista.

Dois deles sendo questionários desenvolvidos por autores de dois estudos $^{17,23}$ e o Perceived Sexual Distress Scale-Hindi (PSDS-H) que oferece a possibilidade de ser aplicado das duas formas, entrevista ou auto administrado. Essa prevalência da utilização de instrumentos auto administrados, pode ser justificada pelo fato de que a sexualidade ainda é um tabu para muitas pessoas e pode gerar um certo constrangimento no momento de responder as perguntas durante uma entrevista.

As propriedades de medida de um instrumento, como validade e confiabilidade, são importantes para determinar o grau em que os resultados de um questionário ou entrevista podem ser confiáveis para representar a realidade dos desfechos para os quais os instrumentos são propostos a medir. ${ }^{9}$

Na presente revisão, os instrumentos já validados para população com lesão medular são: Questionário de Sexualidade Humana na Lesão Medular, Sexual Adjustment Questionnaire (SAQ), Sexual Attitude and Information Questionnaire (SAIQ), Perceived Sexual Distress Scale (PSDS) e as versões de auto-relato do International SCI Male Sexual Function e do Female Sexual and Reproductive Function (SR-iSCl-sexual function).

Esses questionários não são limitados a apenas um gênero, podendo ser utilizados para avaliação de homens e mulheres e permitem avaliar desfechos como satisfação, desempenho e ajuste sexual, assim como relação com o parceiro, aconselhamento sexual, entre outros.

Foi observado com os estudos incluídos, ${ }^{6,7,18-21}$ que investigaram propriedades de medida, que elementos da confiabilidade foram definidos para todos os instrumentos, entretanto apenas dois estudos ${ }^{18,20}$ aprofundaram na validade. Foram observados valores satisfatórios de confiabilidade para todos os instrumentos investigados.

Isso implica que os instrumentos Male Sexual Quotient (MSQ), Sexual Health Inventory for Men (SHIM), Sexual Adjustment Questionnaire (SAQ), Sexual Attitude and Information Questionnaire (SAIQ), Perceived Sexual Distress Scale" (PSDS), International SCI male sexual function e Female sexual and reproductive function (SR-iSClsexual function) podem ser aplicados para indivíduos com lesão medular.

Dos seis estudos metodológicos encontrados na presente revisão, quatro instrumentos são específicos para avaliação da sexualidade de indivíduos com lesão medular e dois são instrumentos genéricos, ou seja, instrumentos que a princípio foram desenvolvidos para avaliação da sexualidade da população em geral, e posteriormente foram validados para população com lesão medular. A vantagem da utilização de instrumentos específicos é que as particularidades da condição de saúde são abordadas.

No caso da lesão medular, o posicionamento, controle de tronco, ausência ou presença de sensibilidade e diferentes mecanismos fisiológicos influenciam na resposta sexual, o que não é abordado por questionários genéricos.

A disponibilidade de instrumentos para avaliação da saúde sexual é muito importante para uma boa prática clínica, pois permite que os 
profissionais da área da saúde avaliem e intervenham de forma eficaz, atingindo os melhores padrões de avaliação e intervenção, e assim, melhorem a qualidade de vida dessa população. A utilização dos questionários na avaliação clínica permite que o paciente se auto avalie em relação aos seus componentes físicos, emocionais e funcionais da sua experiência sexual e repasse essas informações da melhor forma para o profissional que for avaliá-lo.

Embora um número satisfatório de estudos e instrumentos tenham sido coletados para realização desta revisão sistemática, algumas limitações foram encontradas, como a falta de detalhes relacionados aos escores dos instrumentos, informações detalhadas sobre os instrumentos que foram desenvolvidos pelos próprios autores, e propriedades de medida incompletas poucos descritas em alguns casos.

\section{CONCLUSÃO}

Esta revisão identificou um total de 28 instrumentos que foram utilizados na avaliação da sexualidade em indivíduos com lesão medular, porém, alguns não são específicos para esse fim. Os estudos de avaliação das propriedades de medida para instrumentos específicos de sexualidade na população com lesão medular, são poucos, apenas sete desses instrumentos foram validados.

Existem instrumentos disponíveis para utilização na prática clínica que viabilizam uma melhor avaliação entre os profissionais da área da saúde e ajudam aos indivíduos com lesão medular a melhorar a satisfação sexual, através de um melhor diálogo com o parceiro, profissionais e conselheiros. Entretanto, dada a natureza multifatorial da sexualidade nessa população, os instrumentos devem ter suas propriedades de medida testadas e comprovadas.

\section{REFERÊNCIAS}

1. Torrecilha LA, Costa BT, Lima FB, Santos SMSS, Souza RB. O perfil da sexualidade em homens com lesão medular. Rev Fisioter Mov. 2014;27(1):39-48. DOI: http://dx.doi.org/10.1590/01035150.027.001.AO04

2. Consortium for Spinal Cord Medicine. Sexuality and reproductive health in adults with spinal cord injury: a clinical practice guideline for health-care professionals. J Spinal Cord Med. 2010;33(3):281336. DOI: http://doi.org/10.1080/10790268.2010.11689709

3. Alexander MS, Biering-Sørensen F, Elliott S, Kreuter M, Sønksen J. International spinal cord injury male sexual function basic data set. Spinal Cord. 2011;49(7):795-8. DOI http://doi.org/10.1038/sc.2010.192

4. Lombardi G, Del Popolo G, Macchiarella A, Mencarini M, Celso M. Sexual rehabilitation in women with spinal cord injury: a critical review of the literature. Spinal Cord. 2010;48(12):842-9. DOI: http://doi.org/10.1038/sc.2010.36

5. 't Hoen LA, Groen J, Scheepe JR, Reuvers S, Diaz DC, Fernández BP, et al. A Quality assessment of patient-reported outcome measures for sexual function in neurologic patients using the consensusbased standards for the selection of health measurement instruments checklist: a systematic review. Eur Urol Focus. 2017;3(4-5):444-456. http://doi.org/10.1016/j.euf.2016.06.009

6. Miranda EP, Gomes CM, Bessa J Jr, Najjar Abdo CH, Suzuki Bellucci $\mathrm{CH}$, Castro Filho JE, et al. Evaluation of Sexual Dysfunction in Men With Spinal Cord Injury Using the Male Sexual Quotient. Arch Phys Med Rehabil. 2016;97(6):947-52. DOI: http://doi.org/10.1016/j.apmr.2016.01.005

7. New PW, Currie KE. Development of a comprehensive survey of sexuality issues including a self-report version of the International Spinal Cord Injury sexual function basic data sets. Spinal Cord. 2016;54(8):584-91. DOI: http://doi.org/10.1038/sc.2015.216
8. Khak M, Hassanijirdehi M, Afshari-Mirak S, Holakouie-Naieni K, Saadat S, Taheri T, et al. Evaluation of Sexual Function and Its Contributing Factors in Men With Spinal Cord Injury Using a SelfAdministered Questionnaire. Am J Mens Health. 2016;10(1):24-31. DOI: http://doi.org/10.1177/1557988314555122

9. Abramson CE, McBride KE, Konnyu KJ, Elliott SL; SCIRE Research Team. Sexual health outcome measures for individuals with a spinal cord injury: a systematic review. Spinal Cord. 2008;46(5):320-4. DOI: http://doi.org/10.1038/sj.sc.3102136

10. Cobo Cuenca Al, Sampietro-Crespo A, Virseda-Chamorro $M$, Martín-Espinosa N. Psychological impact and sexual dysfunction in men with and without spinal cord injury. J Sex Med. 2015;12(2):436-44. DOI: http://doi.org/10.1111/jsm.12741

11. Kreuter M, Siösteen A, Biering-Sørensen F. Sexuality and sexual life in women with spinal cord injury: a controlled study. J Rehabil Med. 2008;40(1):61-9. DOI: http://doi.org/10.2340/165019770128

12. Siösteen A, Lundqvist C, Blomstrand C, Sullivan L, Sullivan M. Sexual ability, activity, attitudes and satisfaction as part of adjustment in spinal cord-injured subjects. Paraplegia. 1990;28(5):285-95. DOI: http://doi.org/10.1038/sc.1990.38

13. New PW. Sexual abuse in people with spinal cord damage. J Spinal Cord Med. 2019:1-8. DOI: http://doi.org/10.1080/10790268.2019.1621730

14. Harrison J, Glass CA, Owens RG, Soni BM. Factors associated with sexual functioning in women following spinal cord injury. Paraplegia. 1995;33(12):687-92. http://doi.org/10.1038/sc.1995.144

15. Akman RY, Coşkun Çelik E, Karataş M. Sexuality and sexual dysfunction in spinal cord-injured men in Turkey. Turk J Med Sci. 2015;45(4):758-61. DOI: http://doi.org/10.3906/sag-1406-61

16. Kreuter M, Sullivan $M$, Siösteen $A$. Sexual adjustment after spinal cord injury-comparison of partner experiences in pre and post injury relationships. Paraplegia. 1994;32(11):759-70. DOI: http://doi.org/10.1038/sc.1994.122

17. Sharma SC, Singh R, Dogra R, Gupta SS. Assessment of sexual functions after spinal cord injury in Indian patients. Int J Rehabil Res. 2006;29(1):17-25.

DOI: http://doi.org/10.1097/01.mrr.00001855947.56810.fc

18. Merghati-Khoei E, Maasoumi R, Rahdari F, Bayat A, Hajmirzaei S, Lotfi S, et al. Psychometric properties of the Sexual Adjustment Questionnaire (SAQ) in the Iranian population with spinal cord injury. Spinal Cord. 2015;53(11):807-10. DOI: http://doi.org/10.1038/sc.2015.69

19. Brockway JA, Steger JC. Sexual attitude and information questionnaire: reliability and validity in a spinal cord injured population. Sex Disabil. 1980;3(4):49-60. DOI: https://doi.org/10.1007/BF01102464

20. Paneri V, Aikat R. Development of the 'Perceived Sexual Distress Scale-Hindi' for measuring sexual distress following spinal cord injury. Spinal Cord. 2014;52(9):712-6. DOI: http://doi.org/10.1038/sc.2014.83

21. Merghati Khoei E, Norouzi Javidan A, Abrishamkar M, Yekaninejad MS, Chaibakhsh S, Emami-Razavi SH, et al. Development, validity and reliability of sexual health measures for spinal cord injured patients in iran. Int J Fertil Steril. 2013;7(2):82-7.

22. Cardoso FL, Savall AC, Mendes AK. Self-awareness of the male sexual response after spinal cord injury. Int J Rehabil Res. 2009;32(4):294-300. http://doi.org/10.1097/MRR.0b013e3283106ab7

23. Julia PE, Othman AS. Barriers to sexual activity: counselling spinal cord injured women in Malaysia. Spinal Cord. 2011;49(7):791-4. DOI: http://doi.org/10.1038/sc.2011.4

24. Abdo $\mathrm{CH}$. The male sexual quotient: a brief, self-administered questionnaire to assess male sexual satisfaction. J Sex Med. 2007;4(2):382-9. DOI: $6109.2006 .00414 . x$ 
Quadro 1. Características dos estudos, amostras e instrumentos de avaliação

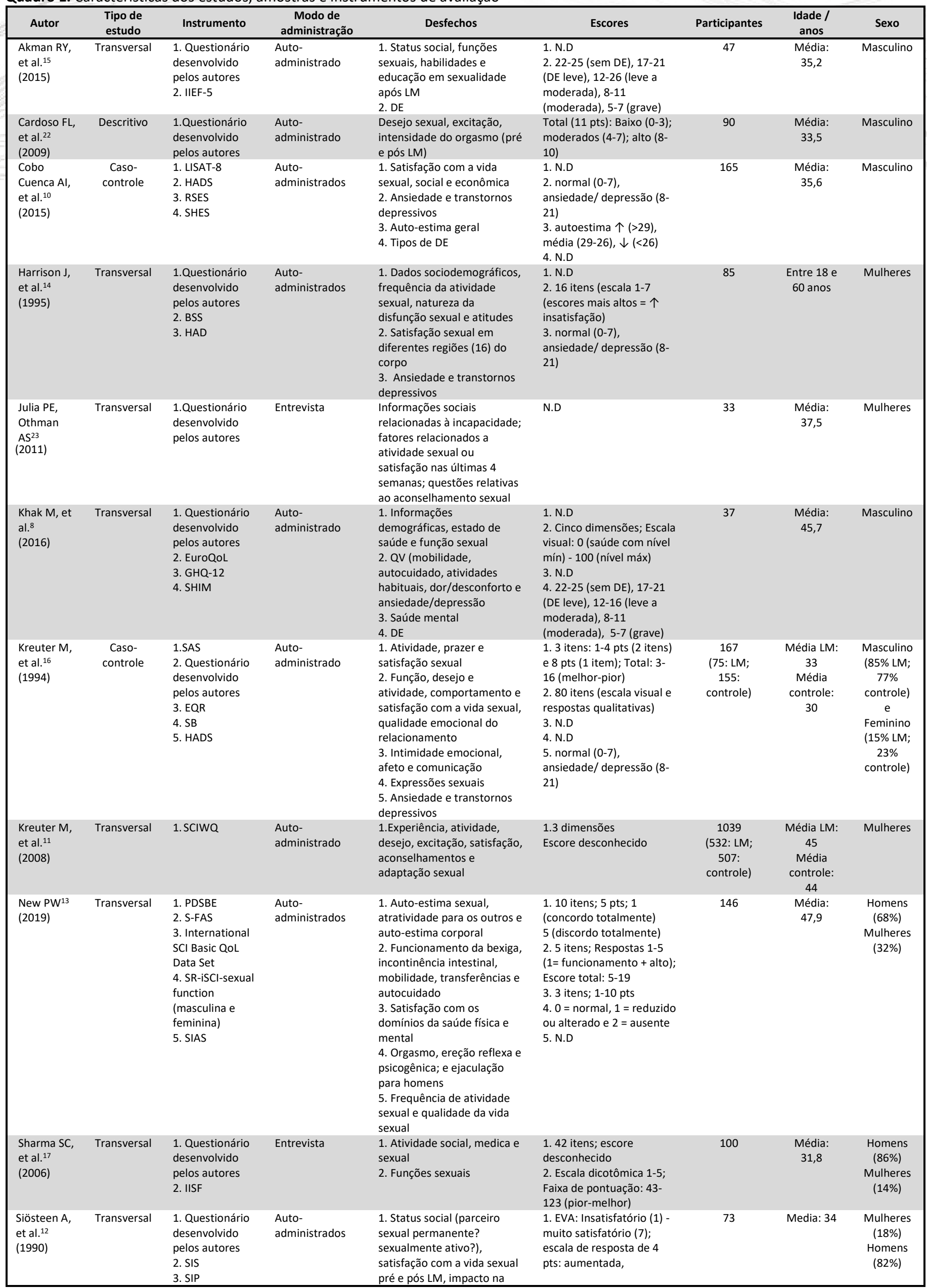


Silva Moura ACML, Costa PHV, Polese JC. Instrumentos de avaliação da sexualidade em homens e mulheres após a lesão medular: uma revisão sistemática

\begin{tabular}{|c|c|c|c|c|c|c|c|c|}
\hline & & $\begin{array}{l}\text { 4. MACL } \\
\text { 5. HAD }\end{array}$ & & $\begin{array}{l}\text { sexualidade, limitações } \\
\text { físicas e deficiências e } \\
\text { capacidade de comunicação } \\
\text { com o parceiro sexual } \\
\text { 2. Interesse em sexualidade } \\
\text { e satisfação sexual } \\
\text { 3. Estado de saúde } \\
\text { 4. Bem-estar mental } \\
\text { 5. Ansiedade e transtornos } \\
\text { depressivos }\end{array}$ & $\begin{array}{l}\text { inalterada, diminuída e } \\
\text { ausente } \\
\text { 2. } 6 \text { itens (0-3); 0-18 (pior } \\
\text { melhor) } \\
\text { 3. N.D } \\
\text { 4. N.D } \\
\text { 5. normal (0-7), } \\
\text { ansiedade/ depressão (8- } \\
\text { 21) }\end{array}$ & & & \\
\hline $\begin{array}{l}\text { Torrecilha } \\
\text { LA, et al. }^{1} \\
(2014)\end{array}$ & Transversal & 1. QSH-LM & $\begin{array}{l}\text { Auto- } \\
\text { administrado }\end{array}$ & $\begin{array}{l}\text { 1.Informações pessoais, } \\
\text { prática, orientação, } \\
\text { satisfação e respostas } \\
\text { sexuais }\end{array}$ & $\begin{array}{l}1.67 \text { questões abertas } \\
\text { (qualitativas e } \\
\text { quantitativas) e fechadas }\end{array}$ & 36 & $\begin{array}{l}\text { Média: } \\
36,6\end{array}$ & Masculino \\
\hline
\end{tabular}

IIEF-5 = International Index of Erectile Function-5; DE = Disfunção Erétil; N.D = Não descrito; $L M=$ Lesão Medular; EuroQoL = Euro Quality of Life questionnaire; GHQ-12 = General Health Questionnaire; SHIM = Sexual Health Inventory for Men; QV = Qualidade de vida; LISAT-8 = Fugl-Meyer Life Satisfaction Questionnaire; HADS = Hospital Anxiety and Depression Scale; RSES = Rosenber Seltion Qualty Functional Ability Scale; SIAS = Sexual Interest. Activity and Satisfaction scale; $B S S=B$ Body Satisfaction Scale

Quadro 2. Características dos estudos de propriedades de medidas dos instrumentos

\begin{tabular}{|c|c|c|c|c|c|c|c|c|}
\hline Autor & Participantes & Sexo & $\begin{array}{l}\text { Instrumento } \\
\text { de avaliação }\end{array}$ & $\begin{array}{c}\text { Modo de } \\
\text { administração }\end{array}$ & $\begin{array}{l}\text { Desfechos } \\
\text { avaliados }\end{array}$ & $\begin{array}{l}\text { Faixa de } \\
\text { Pontuações }\end{array}$ & Confiabilidade & Validade \\
\hline $\begin{array}{l}\text { Brockway JA, } \\
\text { Steger JC }{ }^{19} \\
(1980)\end{array}$ & 34 & $\begin{array}{c}\text { Homens } \\
(71 \%) \\
\text { Mulheres } \\
(29 \%)\end{array}$ & SAIQ & $\begin{array}{c}\text { Auto- } \\
\text { administrado }\end{array}$ & $\begin{array}{l}\text { Informação } \\
\text { Sexual, } \\
\text { aceitação, } \\
\text { preocupações } \\
\text { sexuais e não } \\
\text { sexuais. }\end{array}$ & $\begin{array}{l}37 \text { questões (4 subescalas) } \\
\text { Pontuação: } 20-132 \\
\text { (melhor-pior): subescala 1: 1-18 } \\
\text { (pior-melhor): subescala 2: 6-36 } \\
\text { (pior-melhor): subescala 3: 8-42 } \\
\text { (pior-melhor): subesala 4: 6-36 } \\
\text { (melhor pior) }\end{array}$ & 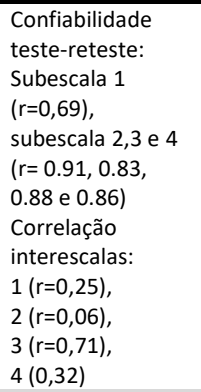 & N.D \\
\hline $\begin{array}{l}\text { Merghati } \\
\text { Khoei E, } \\
\text { et al. }{ }^{21} \\
(2013)\end{array}$ & 40 & $\begin{array}{l}\text { Homens } \\
(80 \%) \\
\text { Mulheres } \\
(20 \%)\end{array}$ & SHM & $\begin{array}{c}\text { Auto- } \\
\text { administrado }\end{array}$ & $\begin{array}{l}\text { Vida social } \\
\text { relacionada ao } \\
\text { sexo, ajuste } \\
\text { sexual, } \\
\text { atividades } \\
\text { sexuais, } \\
\text { satisfação com } \\
\text { o parceiro e } \\
\text { funções sexuais }\end{array}$ & $\begin{array}{l}65 \text { questões } \\
\text { Pontuação 1-5 = } 1 \text { (discordo } \\
\text { totalmente), } 2 \text { (discordo), } 3 \\
\text { (indeciso), } 4 \text { (concordo) e } 5 \\
\text { (concordo totalmente) } \\
\text { EVA = } 1 \text { (muito ruim) } \\
7 \text { (muito bom) }\end{array}$ & $\begin{array}{l}\text { Consistência } \\
\text { interna ( } \alpha \\
\text { Cronbach): 0,73 } \\
\text { (atividade sexual) } \\
\text { a 0,90 (ajuste } \\
\text { sexual) = } \\
\text { aceitável } \\
\text { Confiabilidade } \\
\text { teste-reteste } \\
\text { (ICC): } 0,653 \\
\text { (função sexual } \\
\text { feminina) a 0,836 } \\
\text { (atividade sexual) } \\
\text { = satisfatória }\end{array}$ & N.D \\
\hline $\begin{array}{l}\text { Merghati } \\
\text { Khoei E, et } \\
{\text { al. }{ }^{18}}_{(2015)}\end{array}$ & 200 & $\begin{array}{l}\text { Homens } \\
(73 \%) \\
\text { Mulheres } \\
(27 \%)\end{array}$ & SAQ & $\begin{array}{c}\text { Auto- } \\
\text { administrado }\end{array}$ & $\begin{array}{l}\text { Vida social, } \\
\text { ajustes, } \\
\text { atividades, } \\
\text { desempenho e } \\
\text { fantasias } \\
\text { sexuais; e } \\
\text { satisfação com } \\
\text { o parceiro }\end{array}$ & $\begin{array}{l}11 \text { questões } \\
\text { Pontuação: } 1 \text { (concordo } \\
\text { completamente) } \\
5 \text { (discordo completamente) }\end{array}$ & $\begin{array}{l}\text { Consistência } \\
\text { interna ( } \alpha \\
\text { Cronbach): 0,77 = } \\
\text { Acima dos limites } \\
\text { aceitáveis } \\
\text { Confiabilidade } \\
\text { teste-reteste } \\
\text { (ICC): } 0,72= \\
\text { Confiável }\end{array}$ & $\begin{array}{l}\text { Conteúdo: } \\
\text { gramática, } \\
\text { redação e } \\
\text { alocação de } \\
\text { itens = } \\
\text { apropriados } \\
\text { Face: todos os } \\
\text { participantes } \\
\text { relataram que } \\
\text { não tiveram } \\
\text { problemas em } \\
\text { ler e entender } \\
\text { os itens }\end{array}$ \\
\hline $\begin{array}{l}\text { Miranda } \\
\text { EP, et al. }{ }^{6} \\
(2016)\end{array}$ & 295 & Homens & $\begin{array}{l}\text { MSQ (1) + } \\
\text { SHIM (2) }\end{array}$ & $\begin{array}{c}\text { Auto- } \\
\text { administrado }\end{array}$ & $\begin{array}{l}\text { 1. Função e } \\
\text { satisfação } \\
\text { sexual (desejo, } \\
\text { confiança, } \\
\text { desempenho e } \\
\text { FE) } \\
\text { 2. FE }\end{array}$ & $\begin{array}{l}\text { 1. } 10 \text { questões; Pontuação = } 0 \\
\text { (nunca) }-5 \text { (sempre) } \\
\text { 2. } 5 \text { questões; Respostas = } 0 \\
\text { (pior função sexual) } \\
5 \text { (melhor função sexual) }\end{array}$ & $\begin{array}{l}\text { Coeficiente de } \\
\text { correlação de } \\
\text { Pearson entre os } \\
\text { dois } \\
\text { instrumentos ( } r= \\
1670.826,95 \% \text { Cl } \\
0.779-0.864 \text { ) = } \\
\text { Forte } \\
\text { Correlação entre } \\
\text { as questões dos } \\
\text { dois } \\
\text { instrumentos } \\
\text { relacionadas a } \\
\text { ereção: } r=170 \\
0,844, \text { IC } 95 \% \\
0,802-0,878= \\
\text { Forte }\end{array}$ & N.D \\
\hline
\end{tabular}


Silva Moura ACML, Costa PHV, Polese JC. Instrumentos de avaliação da sexualidade em homens e mulheres após a lesão medular: uma revisão sistemática

\begin{tabular}{|c|c|c|c|c|c|c|c|c|}
\hline $\begin{array}{l}\text { New PW, } \\
\text { Currie KE } \\
(2016)\end{array}$ & 154 & $\begin{array}{c}\text { Homens } \\
(65 \%) \\
\text { Mulheres } \\
(34 \%) \\
\text { Transgênero } \\
(1 \%)\end{array}$ & $\begin{array}{c}\text { SR-ISCI- } \\
\text { SEXUAL } \\
\text { FUNCTION } \\
\text { DATA SETS }\end{array}$ & $\begin{array}{c}\text { Auto- } \\
\text { administrado }\end{array}$ & $\begin{array}{l}\text { Gênero, } \\
\text { orientação } \\
\text { sexual, } \\
\text { habilidades } \\
\text { funcionais; } \\
\text { condições } \\
\text { secundárias e } \\
\text { complicações } \\
\text { relacionadas à } \\
\text { DF, educação } \\
\text { sexual, auto- } \\
\text { estima sexual e } \\
\text { corporal, } \\
\text { interesse sexual, } \\
\text { desejo, } \\
\text { atividades, } \\
\text { satisfação e } \\
\text { problemas de } \\
\text { relacionamento } \\
\text { e abuso sexual }\end{array}$ & $\begin{array}{l}\text { Itens de função sexual: } \\
0=\text { normal, } 1 \text { = reduzido ou } \\
\text { alterado e } 2 \text { = ausente } \\
\text { Outros itens: } 0 \text { (problema não } \\
\text { experimentado/insignificante) } \\
3 \text { (problema } \\
\text { significativo/crônico) }\end{array}$ & 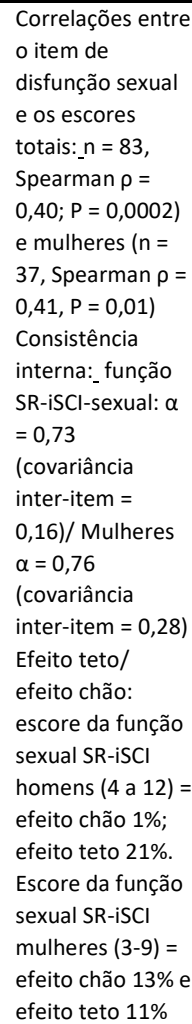 & N.D \\
\hline $\begin{array}{l}\text { Paneri V, } \\
\text { Aikat R } 20 \\
\text { (2014) }\end{array}$ & 30 & $\begin{array}{l}\text { Homens } \\
(93 \%) \\
\text { Mulheres } \\
(7 \%)\end{array}$ & PSDS-H & $\begin{array}{c}\text { Auto- } \\
\text { administrado e } \\
\text { entrevista }\end{array}$ & $\begin{array}{l}\text { Sofrimento } \\
\text { percebido } \\
\text { devido a } \\
\text { disfunção sexual }\end{array}$ & $\begin{array}{l}38 \text { questões (5 pts) } \\
\text { Pontuação total: 0-152 } \\
\text { (melhor-pior) }\end{array}$ & $\begin{array}{l}\text { Consistência } \\
\text { interna ( } \alpha \\
\text { Cronbach): 0,965 } \\
\text { Confiabilidade } \\
\text { teste-reteste: } \\
0,647 \\
\text { Correlação inter- } \\
\text { escalas: } 0,02 \text { - } \\
0,86\end{array}$ & $\begin{array}{l}\text { Conteúdo: } \\
\text { Questões 9, } \\
\text { 11, 13, 14 e } \\
21: \text { IVC }<0,42 \\
(p=0,05) \\
\text { Outros itens: } \\
\text { IVC }=1,0 \text { ( } p= \\
0,05)\end{array}$ \\
\hline
\end{tabular}

$M S Q=$ Male Sexual Quotient; SHIM = Sexual Health Inventory for Men; FE = Função erétil; SHM = Sexual health measures; SAQ = Sexual Adjustment Questionnaire; SAIQ = Sexual Attitude and Information Questionnaire; N.D = Não descrito; PSDS-H = Perceived Sexual Distress Scale-Hindi; IVC = Índice de validade de conteúdo 